\title{
Between the galaxies
}

\section{from M.G. Edmunds}

Who could ask for a better probe of the material between the galaxies than that provided by quasars? Situated at a range of distances, some very great, the quasars give off plenty of radiation to allow the absorption spectrum of the intervening gas to be observed on Earth. The use on the largest telescopes of very sensitive light detectors capable of working with high wavelength resolution has led over the past few years to rather good data on the absorption spectra of several quasars. Particularly successful has been a detector developed at University College, London. Data on six quasars obtained with this instrument have been used to deduce the existence and properties of clouds of intergalactic material (Sargent, Young, Boksenberg \& Tytler Astrophys. J. Suppl. Ser., 42, 41; 1980). These gas clouds are thought to be pure remnants from the initial 'Big-Bang'.

A formidable array of statistical tests on the spectra identified two main sources of absorption. The authors concentrate their attention on the Lyman- $\alpha$ line of hydrogen which normally appears in the ultraviolet at $1,216 \AA$, but the expansion of the Universe redshifts this line into the optical region as observed from Earth provided the absorber is far enough away. The Lyman- $\alpha$ emission (and absorption) intrinsic to the quasar and its surroundings are of course at the highest redshift in the spectrum, but many other absorptions almost certainly attributable to hydrogen appear at redshifts smaller than that of the quasar's Lyman- $\alpha$. In interpreting the origin of these lines, the observed family of lines arising from three-times ionised carbon (CIV) is important. Of the two distinct types of absorbing region which seem to be present, the first kind gives rise to these CIV lines and simply represents the outer regions of galaxies through which the light from the quasar passes on its way to us. There is good evidence that the outer parts of galaxies do contain sufficient gas at the right excitation state to provide the absorption, as the same CIV line is seen in the outer halo of our own Galaxy when looking at the ultraviolet spectra of sources nearby, but outside, our own system (for example the International Ultraviolet Explorer Satellite observations of sources in the Magellanic Clouds reported by Savage and de Boer Astrophys. J. Lett. 230, L77; 1979).

But the other type of absorbing region which gives rise to Lyman- $\alpha$ but no CIV is new, and is suggested to represent true intergalactic clouds. The physical parameters of these clouds are inevitably uncertain but the proposed sizes are $2 \times 10^{7}$

M.G. Edmunds is in the Department of Astronomy and Applied Mathematics, University College, Cardiff.

$0028-0836 / 80 / 12213-03 \$ 01.00$ solar masses and a typical linear dimension of $30 \mathrm{kpc}$. This extent is about the same as a medium-sized galaxy, but the density of the cloud is very much smaller, as that of a galactic mass will be more than $10^{11}$ solar masses. The gas in the clouds seems to contain no (or very few) heavy elements that is, nothing more complex than helium, as no absorption lines of heavy elements are seen at the wavelengths expected on the basis of the redshifts of the hydrogen lines. This implies that the gas is a pure remnant from the Big-Bang initial synthesis which leads to production of hydrogen and helium, but very little else. This contrasts with the gas in galaxies (and their halos) which has been contaminated with heavy elements produced by nuclear reactions in stars and their supernovae explosions. From the relative distances of the clouds as implied by their redshifts, the clouds are not clustered together in space in the way that galaxies are. This is good evidence that they represent genuine intergalactic clouds, and are not associated in any way with existing galaxies.

The discovery of a very primitive kind of object in the Universe is an interesting one, but it must be pointed out that the clouds may have comparatively little influence over the evolution of the Universe as a whole. Their number per unit volume of space at present is only about five per cubic megaparsec, fewer than the observed galaxies and very much less significant in terms of mass. Thus these new objects certainly do not supply sufficient mass to close the Universe and make it eventually re-collapse on itself - a role often proposed for intergalactic material.

Sargent et al.'s analysis of the thermal state of the cloud gas implies that the clouds are too hot to be bound by their selfgravitation, and must be confined by an even hotter tenuous intergalactic gas. But it is significant that such a gas could still not be hot enough to provide enough $\mathrm{X}$-ray emission to explain the diffuse background of $\mathrm{X}$ rays observed in space. (The initial results from the Einstein observatory imply that much of this background may anyway be due to the quasars themselves). The allowed cloud parameters are apparently rather tightly constrained - in these physical conditions a rather bigger or smaller mass than is suggested gives theoretical estimates that imply they would either collapse or evaporate. It is perhaps surprising that such fragile clouds have managed to survive to the present day presumably they represent a small fraction of clouds of a whole range of sizes which formed early on in the evolution of the Universe. The other clouds either collapsed (and perhaps combined) to give galaxies and quasars, or else dispersed to become the diffuse intergalactic medium.
Can we see any of these primitive clouds near our own Galaxy? The International Ultraviolet Explorer Satellite provides an opportunity to look at the brightest quasar $3 \mathrm{C} 273$, but as yet no conclusive evidence of suitable Lyman- $\alpha$ absorption has been reported, although the CIV absorbing gas of our own galactic halo is clearly visible. But the number density of the clouds would predict only three clouds in the line of sight to $3 C 273$ - so even a complete failure to detect anything would not be too surprising on statistical grounds.

Undoubtedly this study goes a long way to demonstrating that a large fraction of absorption lines in quasar spectra may be satisfactorily explained as not being intrinsic to the quasars themselves, but due to the outer parts of galaxies and to clouds in the intergalactic medium. Just what fraction of the remaining absorption lines can be attributed to gas driven out of the quasars remains to be seen.

\section{Electrically conducting polymers}

\section{from Paul Calvert}

FOR some time people have been looking for polymers with metallic conductivity or with superconductivity. Sulphur nitride was found in 1975 to be a superconductor at low temperatures and sparked a great deal of research which now seems to have mainly fizzled out. At the moment polyacetylene seems to have potential as a metallic conductor and is the subject of much interest.

For a material to be conducting there must be a supply of charge carriers, electrons or 'holes', and an easy, high mobility path for them to follow through the material. Thus one class of polymeric conductors consists of conjugated chains, with alternating single and double bonds, such that the pi-bonding orbitals overlap to form a path along the chain. Examples of this are sulphur nitride $(-\mathrm{S}=\mathrm{N}-)_{\mathrm{x}}$ and polyacetylene $(-\mathrm{CH}=\mathrm{CH}-)_{x}$. Alternatively, stacked planar aromatic molecules can provide an easy conduction path perpendicular to the molecular plane as in graphite. Hence the interest which developed at one time in the conductivity properties of DNA, where the stacking of the base pairs suggested that the molecule could be a 'one-dimensional' conductor.

Acetylene normally polymerises to a grey, insoluble, infusible powder with all the properties of brick dust, except price. However in 1974 T. Ito, H. Shirakawa and S. Ikeda at Tokyo Institute of Technology (J. Polym. Sci. Chem. 12, 11; 1974) produced thin films by exposing acetylene gas to concentrated solutions of Ziegler

Paul Calvert is a lecturer in the School of Molecular Sciences, University of Sussex. 